\title{
Cooperative Output Regulation of Multiagent Linear Parameter-Varying Systems
}

\author{
Afshin Mesbahi and Javad Mohammadpour Velni \\ Complex Systems Control Laboratory, College of Engineering, The University of Georgia, Athens, GA 30602, USA \\ Correspondence should be addressed to Afshin Mesbahi; mesbahi@uga.edu and Javad Mohammadpour Velni; javadm@uga.edu
}

Received 30 June 2016; Revised 13 November 2016; Accepted 27 November 2016; Published 19 January 2017

Academic Editor: Guangming Xie

Copyright (c) 2017 Afshin Mesbahi and Javad Mohammadpour Velni. This is an open access article distributed under the Creative Commons Attribution License, which permits unrestricted use, distribution, and reproduction in any medium, provided the original work is properly cited.

\begin{abstract}
The output regulation problem is examined in this paper for a class of heterogeneous multiagent systems whose dynamics are governed by polytopic linear parameter-varying (LPV) models. The dynamics of the agents are decoupled from each other but the agents' controllers are assumed to communicate. To design the cooperative LPV controllers, analysis conditions for closed-loop system are first established to ensure stability and reference tracking. Then, the LPV control synthesis problem is addressed, where the offline solution to a time-varying Sylvester equation will be used to determine and update in real time the controller state-space matrices. Two numerical examples will be finally given to demonstrate the efficacy of the proposed cooperative design method.
\end{abstract}

\section{Introduction}

We have witnessed in the recent years a large number of studies devoted to the area of multiagent systems coordination and control due to their vast applications in biological systems, traffic networks, robotics, and unmanned vehicles, among many other applications (see, e.g., [1-3] and references therein). In particular, the problem of output regulation for multiagent linear systems has been largely investigated (see, e.g., $[4-7])$.

In many practical multiagent systems, agents often exhibit time-varying dynamics due to the variation in some endogenous or exogenous parameters, which are bounded and measurable in real time. Such physical systems can be modeled and controlled, in principle, by means of the well developed tools from linear parameter-varying (LPV) systems theory [8-11]. There has been a very limited work dedicated to multiagent LPV systems. In this paper, we formulate and address the design of output regulators for multiagent polytopic LPV systems.

The output synchronization problem of multiagent systems has been studied for agents represented by identical LPV models or bilinear models with noisy measurements $[12,13]$. In these studies, the controllers are designed based on the dissipativity theory, while it is assumed that each agent has access to the varying parameters of all other agents. The output synchronization problem has been investigated for heterogenous multiagent LPV systems [14]. The full state feedback controller was considered to solve a consensus problem for homogeneous and heterogenous multiagent LPV systems with affine dependency on LPV parameters, a.k.a. scheduling variables [15].

The main contribution of this paper is in the design of a cooperative output feedback controller to solve the output regulation problem for multiagent polytopic LPV systems. The parameter-varying controllers are designed under the assumption that each agent is decoupled from others, while each agent's controller is able to communicate with other controllers and send/receive states to/from others. A sufficient condition is first established to ensure that the output regulation problem is solvable for a multiagent LPV system. This condition is then converted to the so-called Sylvester equation with time-varying coefficients. A time-varying Sylvester equation can be solved by using, for example, gradient-based recurrent neural networks; however, there always exists an error in the calculated solution [16, 17]. A special class of recurrent neural networks has been proposed in [16] to solve time-varying Sylvester equations in real time leading to a zero estimation error. The proposed recurrent neural network also has limitations for real time implementation since the 
estimation error does not converge to zero in finite time [18]. By employing a sign-bipower activation function and $\mathrm{Li}$ activation function, the estimation error converges to zero in a finite time $[18,19]$, but the estimation error is conservative [20]. In this paper, a condition is obtained for the case, where time-varying coefficients of the Sylvester equation have polytopic structures. In this case, a simple sufficient condition is determined to guarantee the existence of the LPV controller when the models of multiagent LPV system have a polytopic structure.

The remainder of this paper is structured as follows. Multiagent systems described by LPV models are introduced in Section 2. This section also gives a brief overview of the basic mathematical tools from graph theory and contains the problem statement for the cooperative output regulation problem of a multiagent LPV system. The main results are presented in Section 3, where a condition is provided for the design of a controller solving the cooperative output regulation problem. In Section 4, the obtained conditions are simplified for multiagent LPV systems with a polytopic structure. In Section 5, the efficacy of the proposed control design approach is examined by means of two numerical examples, and finally, concluding remarks are made in Section 6.

Notation. Throughout this paper, we assume that $\mathbb{R}, \mathbf{I}, \mathbf{A}^{T}$, $\operatorname{diag}(\mathbf{A}, \mathbf{B}), \operatorname{col}(\mathbf{A}, \mathbf{B}), \operatorname{vec}(\mathbf{A}), \mathbf{A} \otimes \mathbf{B}$, and $\mathbf{A} \oplus \mathbf{B}$ denote the set of real numbers, the identity matrix of appropriate dimension, the transpose of $\mathbf{A}$, the block diagonal matrix with diagonal elements $\mathbf{A}$ and $\mathbf{B},\left[\mathbf{A}^{T}, \mathbf{B}^{T}\right]^{T}$, the vectorization of $\mathbf{A}$, the Kronecker product of $\mathbf{A}$ and $\mathbf{B}$, and the Kronecker sum of $\mathbf{A} \in \mathbb{R}^{n \times n}$ and $\mathbf{B} \in \mathbb{R}^{m \times m}$ defined as $\mathbf{A} \oplus \mathbf{B}=\mathbf{A} \otimes \mathbf{I}_{m}+\mathbf{I}_{n} \otimes \mathbf{B}$.

\section{Preliminaries and Problem Statement}

In this section, the dynamics of agents and the problem under study will be described.

2.1. Communication Structure. We use a directed or undirected graph denoted by $G=(\mathscr{V}, \mathscr{E})$ to model the communication among the agents, where $\mathscr{V}=\{1, \ldots, N\}$ denotes the set of nodes and $\mathscr{E} \subseteq \mathscr{V} \times \mathscr{V}$ denotes the edge set. In an undirected graph, $(j, i) \in \mathscr{E}$ implies that $(i, j) \in \mathscr{E}$. Agent $i$ is said to have access to the information of agent $j$ when $(j, i) \in \mathscr{E}$, in which case agent $j$ is also called the neighbor of agent $i$. A directed path from node $i$ to node $j$ is a sequence of ordered edges of the form $(i, n),(n, m), \ldots,(r, j)$. A graph has a directed spanning tree rooted at node $i$ if there is a directed path from the node $i$ to all other nodes. Nonnegative matrix $\mathscr{A}=\left[a_{i j}\right] \in \mathbb{R}^{N \times N}$ is a weighted adjacency matrix of digraph $G$ if $a_{i i}=0$ and $a_{i j}>0$ for $(i, j) \in \mathscr{E}$. Finally, Laplacian matrix $L=\left[l_{i j}\right] \in \mathbb{R}^{N \times N}$ of digraph $G$ is defined as $l_{i i}=\sum_{i=1, i \neq j}^{N} a_{i j}$ and $l_{i j}=-a_{i j}>0$ for $i \neq j$.

2.2. Mathematical Representation of the Agents. We consider that each of the $N$ agents in the multiagent systems under study is represented by a linear parameter-varying (LPV) model as

$$
\begin{aligned}
\dot{\mathbf{x}}_{k}(t)= & \mathbf{A}_{k}\left(\rho_{k}(t)\right) \mathbf{x}_{k}(t)+\mathbf{B}_{k} \mathbf{u}_{k}(t) \\
& +\mathbf{E}_{k}\left(\rho_{k}(t)\right) \mathbf{d}_{k}(t),
\end{aligned}
$$

$$
\begin{aligned}
\mathbf{y}_{k}(t)= & \mathbf{C}_{k}\left(\rho_{k}(t)\right) \mathbf{x}_{k}(t)+\mathbf{D}_{k} \mathbf{u}_{k}(t) \\
& +\mathbf{F}_{k}\left(\rho_{k}(t)\right) \mathbf{d}_{k}(t), \\
\mathbf{e}_{k}(t)= & \mathbf{C}_{k}^{e}\left(\rho_{k}(t)\right) \mathbf{x}_{k}(t)+\mathbf{D}_{k}^{e} \mathbf{u}_{k}(t) \\
& +\mathbf{F}_{k}^{e}\left(\rho_{k}(t)\right) \mathbf{d}_{k}(t),
\end{aligned}
$$

for $k \in \mathscr{N}=\{1, \ldots, N\}$, where $\mathbf{x}_{k} \in \mathbb{R}^{n_{x_{k}}}$ is the state vector, $\mathbf{y}_{k} \in \mathbb{R}^{n_{y_{k}}}$ is the measurement outputs vector, $\mathbf{e}_{k} \in$ $\mathbb{R}^{n_{e_{k}}}$ is the vector of controlled outputs, for example, tracking error, and $\mathbf{u}_{k} \in \mathbb{R}^{n_{u_{k}}}$ is the vector of control inputs for agent $k$. Also, $\mathbf{d}_{k} \in \mathbb{R}^{n_{d_{k}}}$ denotes the exogenous signals to agent $k$. Unlike the system matrices $\mathbf{B}_{k}, \mathbf{D}_{k}$, and $\mathbf{D}_{k}^{e}$ that are assumed to be constant, the matrices $\mathbf{A}_{k}, \mathbf{C}_{k}, \mathbf{C}_{k}^{e}, \mathbf{E}_{k}, \mathbf{F}_{k}$, and $\mathbf{F}_{k}^{e}$ could in general depend on the time-varying parameters $\rho_{k}(t)$, referred to as "scheduling variables," which are bounded and measurable in real time. We further assume that $\rho_{k}(t) \epsilon$ $\mathscr{P}_{k}$, where $\mathscr{P}_{k}$ denotes a $p_{k}$-dimensional admissible set of scheduling variables. Even in the case that the agents share the same dynamics structure, that is, number of states, inputs, and outputs, the agents can be heterogeneous due to the difference in the measurement of the local scheduling variables $\rho_{k}(t)$. The exogenous signals may represent the disturbance to be rejected or the reference input to be tracked and are assumed to be described by

$$
\dot{\mathbf{d}}_{k}(t)=\mathbf{S}_{k}\left(\rho_{k}(t)\right) \mathbf{d}_{k}(t) .
$$

Remark 1. Without the loss of generality, the system matrices $\mathbf{B}_{k}, \mathbf{D}_{k}$, and $\mathbf{D}_{k}^{e}$ in system (1) are assumed to be parameterindependent. Generally, a system with parameter-varying $\mathbf{B}_{k}$, $\mathbf{D}_{k}$, or $\mathbf{D}_{k}^{e}$ can be transformed into the form (1) by applying a low-pass filter to the control input [21].

2.3. Cooperative Output Regulation Problem for Multiagent $L P V$ Systems. For each agent, we consider a parametervarying controller with the following structure:

$$
\begin{aligned}
\dot{\mathbf{x}}_{k}^{c}(t)= & \mathbf{A}_{k}^{c}\left(\rho_{k}(t)\right) \mathbf{x}_{k}^{c}(t)+\mathbf{B}_{k}^{c}\left(\rho_{k}(t)\right) \mathbf{y}_{k}(t) \\
& +\sum_{k_{i} \in \mathcal{N}_{k}} \mathbf{A}_{k_{i}}^{c}\left(\rho_{k_{i}}(t)\right) \mathbf{x}_{k_{i}}^{c}(t), \\
\mathbf{u}_{k}(t)= & \mathbf{C}_{k}^{c}\left(\rho_{k}(t)\right) \mathbf{x}_{k}^{c}(t)+\mathbf{D}_{k}^{c}\left(\rho_{k}(t)\right) \mathbf{y}_{k}(t),
\end{aligned}
$$

for $\mathcal{N}_{k}=\{i \mid i \in \mathscr{N}$ and $i \neq k\}$, where $\mathbf{x}_{k}^{c}(t)$ is the estimate of the augmented vector of open-loop system states and exogenous signals $\left(\mathbf{x}_{k}^{a}=\operatorname{col}\left(\mathbf{x}_{k}(t), \mathbf{d}_{k}(t)\right)\right)$, that is, $\mathbf{x}_{k}^{c}=\widehat{\mathbf{x}}_{k}^{a}$. The LPV controllers above use the local state estimates $\left(\mathbf{x}_{k}^{c}=\right.$ $\left.\widehat{\mathbf{x}}_{k}^{a}\right)$ and the controller matrices $\mathbf{A}_{k}^{c}, \mathbf{B}_{k}^{c}, \mathbf{A}_{k_{i}}^{c}, \mathbf{C}_{k}^{c}$, and $\mathbf{D}_{k}^{c}$ are to be determined. We aim at designing the controllers of the above structure to ensure that the following two objectives are satisfied.

Objective 1 (internal stability). Assume $\mathbf{u}_{k}(t)=\mathbf{0}$ and $\mathbf{d}_{k}(t)=$ $\mathbf{0}$ for $k \in \mathcal{N}$. For all initial conditions $\mathbf{x}_{k}(0)=\mathbf{x}_{k, 0}, \mathbf{x}_{k}^{c}(0)=$ $\mathbf{x}_{k, 0}^{c}$, and $\rho_{k} \in \mathscr{P}_{k}$,

$$
\begin{aligned}
& \lim _{t \rightarrow \infty} \mathbf{x}_{k}(t)=0, \\
& \lim _{t \rightarrow \infty} \mathbf{x}_{k}^{c}(t)=0,
\end{aligned}
$$

$$
k \in \mathcal{N} .
$$


Objective 2 (output regulation). For all initial conditions $\mathbf{x}_{k}(0)=\mathbf{x}_{k, 0}, \mathbf{x}_{k}^{c}(0)=\mathbf{x}_{k, 0}^{c}, \mathbf{d}_{k}(0)=\mathbf{d}_{k, 0}$, and $\rho_{k} \in \mathscr{P}_{k}$,

$$
\lim _{t \rightarrow \infty} \mathbf{e}_{k}(t)=0, \quad k \in \mathcal{N}
$$

Remark 2. The main contribution of this paper is in the design of an LPV controller that can guarantee the output regulation problem for multiagent LPV systems. A relevant problem to the output regulation problem has been studied from the synchronization point of view in [12-14]. Furthermore, the output regulation problem has been addressed in [15] for systems, in which the state-space matrices are affinely dependent on the scheduling variables.

\section{Cooperative Output Regulation for Multiagent LPV Systems}

In this section, we present the main results of the paper. First, we describe few assumptions imposed on the agents. It is noted that the following assumptions are standard ones that have also been considered in previous relevant studies $[5,14,15,22-24]$.

Assumption 3. The pair $\left(\mathbf{A}_{k}\left(\rho_{k}(t)\right), \mathbf{B}_{k}\right)$ is stabilizable for any $k \in \mathscr{N}$ and $\rho_{k} \in \mathscr{P}_{k}$.

Assumption 4. The exosystem (2) is not asymptotically stable for any $k \in \mathscr{N}$ and $\rho_{k} \in \mathscr{P}_{k}$.

Assumption 5. The pair

$$
\left(\left[\begin{array}{cc}
\mathbf{A}_{k}\left(\rho_{k}(t)\right) & \mathbf{E}_{k}\left(\rho_{k}(t)\right) \\
\mathbf{0} & \mathbf{S}_{k}\left(\rho_{k}(t)\right)
\end{array}\right],\left[\mathbf{C}_{k}\left(\rho_{k}(t)\right) \mathbf{F}_{k}\left(\rho_{k}(t)\right)\right]\right)
$$

is detectable for any $k \in \mathscr{N}$ and $\rho_{k} \in \mathscr{P}_{k}$.

Augmenting the models of agents together results in the following model:

$$
\begin{aligned}
& \dot{\mathbf{x}}(t)=\mathbf{A}(\rho(t)) \mathbf{x}(t)+\mathbf{B u}(t)+\mathbf{E}(\rho(t)) \mathbf{d}(t), \\
& \mathbf{y}(t)=\mathbf{C}(\rho(t)) \mathbf{x}(t)+\mathbf{D} \mathbf{u}(t)+\mathbf{F}(\rho(t)) \mathbf{d}(t), \\
& \mathbf{e}(t)=\mathbf{C}^{e}(\rho(t)) \mathbf{x}(t)+\mathbf{D}^{e} \mathbf{u}(t)+\mathbf{F}^{e}(\rho(t)) \mathbf{d}(t), \\
& \dot{\mathbf{d}}(t)=\mathbf{S}(\rho(t)) \mathbf{d}(t),
\end{aligned}
$$

where $\rho(t)$ is a vector function of $\rho_{k}(t)$, for $k \in \mathcal{N}$, and $\mathbf{A}=$ $\operatorname{diag}\left(\mathbf{A}_{1}, \ldots, \mathbf{A}_{N}\right), \mathbf{B}=\operatorname{diag}\left(\mathbf{B}_{1}, \ldots, \mathbf{B}_{N}\right), \mathbf{E}=\operatorname{diag}\left(\mathbf{E}_{1}, \ldots\right.$, $\left.\mathbf{E}_{N}\right), \mathbf{C}=\operatorname{diag}\left(\mathbf{C}_{1}, \ldots, \mathbf{C}_{N}\right), \mathbf{D}=\operatorname{diag}\left(\mathbf{D}_{1}, \ldots, \mathbf{D}_{N}\right), \mathbf{F}=$ $\operatorname{diag}\left(\mathbf{F}_{1}, \ldots, \mathbf{F}_{N}\right), \mathbf{C}^{e}=\operatorname{diag}\left(\mathbf{C}_{1}^{e}, \ldots, \mathbf{C}_{N}^{e}\right), \mathbf{D}^{e}=\operatorname{diag}\left(\mathbf{D}_{1}^{e}, \ldots\right.$, $\left.\mathbf{D}_{N}^{e}\right), \mathbf{F}^{e}=\operatorname{diag}\left(\mathbf{F}_{1}^{e}, \ldots, \mathbf{F}_{N}^{e}\right), \mathbf{S}=\operatorname{diag}\left(\mathbf{S}_{1}, \ldots, \mathbf{S}_{N}\right), \mathbf{x}(t)=$ $\operatorname{col}\left(\mathbf{x}_{1}(t), \ldots, \mathbf{x}_{N}(t)\right), \mathbf{u}(t)=\operatorname{col}\left(\mathbf{u}_{1}(t), \ldots, \mathbf{u}_{N}(t)\right), \mathbf{y}(t)=$ $\operatorname{col}\left(\mathbf{y}_{1}(t), \ldots, \mathbf{y}_{N}(t)\right), \mathbf{e}(t)=\operatorname{col}\left(\mathbf{e}_{1}(t), \ldots, \mathbf{e}_{N}(t)\right)$, and $\mathbf{d}(t)=$ $\operatorname{col}\left(\mathbf{d}_{1}(t), \ldots, \mathbf{d}_{N}(t)\right)$. Assumption 3 is held if there exists a symmetric positive definite matrix $\mathbf{P}$ and a matrix $\mathbf{K}_{x}$ such that the following matrix inequality problem has a feasible solution [21]:

$$
\mathbf{P}\left(\mathbf{A}(\rho(t))+\mathbf{B K}_{x}\right)^{T}+\left(\mathbf{A}(\rho(t))+\mathbf{B K}_{x}\right) \mathbf{P}<\mathbf{0} .
$$

In addition, Assumption 5 is held if there exist matrices $\mathbf{L}_{1}$, $\mathbf{L}_{2}$ and a symmetric positive definite matrix $\mathbf{P}_{L}$ such that the following matrix inequality has a feasible solution [21]:

$$
\mathbf{A}_{L}^{T}(\rho(t)) \mathbf{P}_{L}+\mathbf{P}_{L} \mathbf{A}_{L}(\rho(t))<\mathbf{0},
$$

where

$$
\begin{aligned}
& \mathbf{A}_{L}(\rho(t))=\left[\begin{array}{cc}
\mathbf{A}(\rho(t)) & \mathbf{E}(\rho(t)) \\
\mathbf{0} & \mathbf{S}(\rho(t))
\end{array}\right]
\end{aligned}
$$

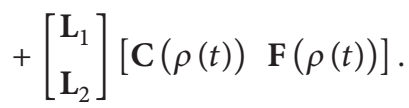

The LPV feedback controller with the structure (12) is applied to the augmented system (7). It is noted that (12) is obtained by augmenting the output feedback controllers with state estimations in (3):

$$
\begin{aligned}
\dot{\mathbf{x}}^{c}(t) & =\mathbf{A}^{c}(\rho(t)) \mathbf{x}^{c}(t)+\mathbf{B}^{c}(\rho(t)) \mathbf{y}(t), \\
\mathbf{u}(t) & =\mathbf{C}^{c}(\rho(t)) \mathbf{x}^{c}(t)+\mathbf{D}^{c}(\rho(t)) \mathbf{y}(t),
\end{aligned}
$$

where $\mathbf{x}^{c}(t)=\operatorname{col}\left(\mathbf{x}_{1}^{c}(t), \ldots, \mathbf{x}_{N}^{c}(t)\right), \mathbf{u}(t)=\operatorname{col}\left(\mathbf{u}_{1}(t), \ldots\right.$, $\left.\mathbf{u}_{N}(t)\right)$, and $\mathbf{y}(t)=\operatorname{col}\left(\mathbf{y}_{1}(t), \ldots, \mathbf{y}_{N}(t)\right)$, while matrices $\mathbf{A}^{c}$, $\mathbf{B}^{\mathrm{c}}, \mathbf{C}^{c}$, and $\mathbf{D}^{c}$ contain controller matrices of all agents. Controller state-space matrices associated with each agent are then calculated based on the matrices $\mathbf{A}^{c}, \mathbf{B}^{c}, \mathbf{C}^{c}$, and $\mathbf{D}^{c}$ as given in Section 4.

The closed-loop system interconnection of the above controller with the augmented system (7) leads to

$$
\begin{aligned}
& {\left[\begin{array}{c}
\dot{\mathbf{x}}(t) \\
\dot{\mathbf{x}}^{c}(t)
\end{array}\right]=\left[\begin{array}{cc}
\mathbf{A}(\rho(t)) & \mathbf{B C}^{c}(\rho(t)) \\
\mathbf{B}^{c}(\rho(t)) \mathbf{C}(\rho(t)) & \mathbf{A}^{c}(\rho(t))+\mathbf{B}^{c}(\rho(t)) \mathbf{D} \mathbf{C}^{c}(\rho(t))
\end{array}\right]\left[\begin{array}{c}
\mathbf{x}(t) \\
\mathbf{x}^{c}(t)
\end{array}\right]+\left[\begin{array}{c}
\mathbf{E}(\rho(t)) \\
\mathbf{B}^{c}(\rho(t)) \mathbf{F}(\rho(t))
\end{array}\right] \mathbf{d}(t) \Longrightarrow} \\
& \dot{\mathbf{x}}_{\mathrm{cl}}(t)=\mathbf{A}_{\mathrm{cl}}(\rho(t)) \mathbf{x}_{\mathrm{cl}}(t)+\mathbf{B}_{\mathrm{cl}}(\rho(t)) \mathbf{d}(t),
\end{aligned}
$$




For the linear time invariant (LTI) case, Lemma 1.4 in [22] relates Objectives 1 and 2. This lemma is extended to the LPV systems in the following lemma.

Lemma 6. Consider Assumption 4 and suppose that the closed-loop system represented by (13) satisfies Objective 1. Then, Objective 2 is satisfied as well if there exists a matrix $\boldsymbol{\Theta}$ satisfying the following set of matrix equations for any admissible $\rho(t)$ :

$$
\begin{array}{r}
\mathbf{A}_{c l}(\rho(t)) \boldsymbol{\Theta}-\boldsymbol{\Theta} \mathbf{S}(\rho(t))+\mathbf{B}_{c l}(\rho(t))=\mathbf{0}, \\
\mathbf{C}_{c l}(\rho(t)) \boldsymbol{\Theta}+\mathbf{D}_{c l}(\rho(t))=\mathbf{0} .
\end{array}
$$

Proof. Equation (14a) is the so-called Sylvester equation. Since Assumption 4 and Objective 1 are held, there exists a unique matrix $\Theta$ which makes the Sylvester equation (14a) have a solution (see [17], which has proven this for time-varying Sylvester equation). The equations in (15) are obtained by substituting $\boldsymbol{\xi}(t) \triangleq \mathbf{x}_{\mathrm{cl}}(t)-\boldsymbol{\Theta d}(t)$ into the closedloop system representation (13) and considering (14a)

$$
\begin{aligned}
& \dot{\xi}(t) \\
& =\mathbf{A}_{\mathrm{cl}}(\rho(t)) \boldsymbol{\xi}(t) \\
& +\left(\mathbf{A}_{\mathrm{cl}}(\rho(t)) \boldsymbol{\Theta}-\boldsymbol{\Theta} \mathbf{S}(\rho(t))+\mathbf{B}_{\mathrm{cl}}(\rho(t))\right) \mathbf{d}(t) \\
& =\mathbf{A}_{\mathrm{cl}}(\rho(t)) \boldsymbol{\xi}(t), \\
& \mathbf{e}(t) \\
& \begin{aligned}
= & \mathbf{C}_{\mathrm{cl}}(\rho(t)) \boldsymbol{\xi}(t) \\
& +\left(\mathbf{C}_{\mathrm{cl}}(\rho(t)) \boldsymbol{\Theta}+\mathbf{D}_{\mathrm{cl}}(\rho(t))\right) \mathbf{d}(t) .
\end{aligned}
\end{aligned}
$$

According to Assumption 3, $\boldsymbol{\xi}(t)$ is asymptotically stable. Since $\lim _{t \rightarrow \infty} \xi(t)=\mathbf{0}$ and the matrix $\Theta$ satisfies (14b), then using (15), $\lim _{t \rightarrow \infty} \mathbf{e}(t)=\mathbf{0}$, and this concludes the proof.

Lemma 6 shows that Objectives 1 and 2 are related. Next lemma establishes this relation through employing the system matrices in (7), exosystem (8), and controller (12) and extends Lemma 1.13 in [22] to the LPV case.

Lemma 7. Suppose that Assumption 4 is held. Also assume that Objective 1 is satisfied by the controller (12) for the closedloop system (13). Objective 2 is satisfied as well if there exist matrices $\Pi, \Gamma(\rho(t))$, and $\Upsilon$ such that

$$
\begin{aligned}
& \mathbf{A}(\rho(t)) \boldsymbol{\Pi}+\mathbf{B} \boldsymbol{\Gamma}(\rho(t))+\mathbf{E}(\rho(t))=\Pi \mathbf{S}(\rho(t)), \\
& \mathbf{A}^{c}(\rho(t)) \mathbf{Y} \\
& +\mathbf{B}^{c}(\rho(t))(\mathbf{C}(\rho(t)) \boldsymbol{\Pi}+\mathbf{D} \boldsymbol{\Gamma}(\rho(t))+\mathbf{F}(\rho(t))) \\
& =\mathbf{Y S}(\rho(t)) \text {, } \\
& \mathbf{C}^{e}(\rho(t)) \boldsymbol{\Pi}+\mathbf{D}^{e} \boldsymbol{\Gamma}(\rho(t))+\mathbf{F}^{e}(\rho(t))=\mathbf{0},
\end{aligned}
$$

Proof. Substituting $\Gamma(\rho(t))=\mathbf{C}^{c}(\rho(t)) \Upsilon$ into (16a) and (16b) results in

$$
\begin{aligned}
& \mathbf{A}(\rho(t)) \boldsymbol{\Pi}+\mathbf{B C}^{c}(\rho(t)) \mathbf{Y}+\mathbf{E}(\rho(t))=\Pi \mathbf{S}(\rho(t)), \\
& \mathbf{B}^{c}(\rho(t)) \mathbf{C}(\rho(t)) \boldsymbol{\Pi}+\mathbf{B}^{c}(\rho(t)) \mathbf{F}(\rho(t)) \\
& \quad+\left(\mathbf{A}^{c}(\rho(t))+\mathbf{B}^{c}(\rho(t)) \mathbf{D C}^{c}(\rho(t))\right) \mathbf{Y} \\
& \quad=\mathbf{Y S}(\rho(t)) \\
& \mathbf{C}^{e}(\rho(t)) \boldsymbol{\Pi}+\mathbf{D}^{e} \mathbf{C}^{c}(\rho(t)) \mathbf{Y}+\mathbf{F}^{e}(\rho(t))=\mathbf{0} .
\end{aligned}
$$

Equations (18a) and (18b) are, respectively, obtained from combining (17a) and (17b) with (17c).

$$
\begin{aligned}
& {\left[\begin{array}{cc}
\mathbf{A}(\rho(t)) & \mathbf{B C}^{c}(\rho(t)) \\
\mathbf{B}^{c}(\rho(t)) \mathbf{C}(\rho(t)) & \mathbf{A}^{c}(\rho(t))+\mathbf{B}^{c}(\rho(t)) \mathbf{D C}^{c}(\rho(t))
\end{array}\right]\left[\begin{array}{l}
\boldsymbol{\Pi} \\
\mathbf{\Upsilon}
\end{array}\right]} \\
& +\left[\begin{array}{l}
\boldsymbol{\Pi} \\
\mathbf{\Upsilon}
\end{array}\right] \mathbf{S}(\rho(t))+\left[\begin{array}{c}
\mathbf{E}(\rho(t)) \\
\mathbf{B}^{c}(\rho(t)) \mathbf{F}(\rho(t))
\end{array}\right]=\mathbf{0}, \\
& {\left[\begin{array}{lll}
\mathbf{C}^{e}(\rho(t)) & \mathbf{D}^{e} \mathbf{C}^{c}(\rho(t))
\end{array}\right]\left[\begin{array}{l}
\boldsymbol{\Pi} \\
\mathbf{\Upsilon}
\end{array}\right]+\mathbf{F}^{e}(\rho(t))=\mathbf{0} .}
\end{aligned}
$$

Define the matrix decomposition $\Theta \triangleq \operatorname{col}(\boldsymbol{\Pi}, \mathbf{\Upsilon})$. Equations (14a) and (14b) are, respectively, obtained by substituting $\Theta$ into (18a) and (18b). Then, according to Lemma 6, Objective 2 is satisfied, and this concludes the proof.

Next, let the state-space matrices of the controller (12) be constructed as follows:

$$
\begin{aligned}
& \mathbf{A}^{c}(\rho(t))=\left[\begin{array}{cc}
\mathbf{A}(\rho(t)) & \mathbf{E}(\rho(t)) \\
\mathbf{0} & \mathbf{S}(\rho(t))
\end{array}\right] \\
& +\left[\begin{array}{l}
\mathbf{B} \\
\mathbf{0}
\end{array}\right]\left[\begin{array}{ll}
\mathbf{K}_{x} & \mathbf{K}_{d}(\rho(t))
\end{array}\right] \\
& +\left[\begin{array}{l}
\mathbf{L}_{1} \\
\mathbf{L}_{2}
\end{array}\right]\left(\left[\begin{array}{ll}
\mathbf{C}(\rho(t)) & \mathbf{F}(\rho(t))
\end{array}\right]\right. \\
& \left.+\mathbf{D}\left[\begin{array}{ll}
\mathbf{K}_{x} & \mathbf{K}_{d}(\rho(t))
\end{array}\right]\right), \\
& \mathbf{B}^{c}(\rho(t))=-\left[\begin{array}{l}
\mathbf{L}_{1} \\
\mathbf{L}_{2}
\end{array}\right], \\
& \mathbf{C}^{c}(\rho(t))=\left[\begin{array}{ll}
\mathbf{K}_{x} & \mathbf{K}_{d}(\rho(t))
\end{array}\right], \\
& \mathbf{D}^{c}(\rho(t))=\mathbf{0},
\end{aligned}
$$

where $\mathbf{K}_{d}(\rho(t)) \triangleq \Gamma(\rho(t))-\mathbf{K}_{x} \boldsymbol{\Pi}$ and matrices $\boldsymbol{\Pi}, \boldsymbol{\Gamma}(\rho(t)), \mathbf{K}_{x}$, and $\operatorname{col}\left(\mathbf{L}_{1}, \mathbf{L}_{2}\right)$ are, respectively, obtained from (16a)-(16b) and by solving the matrix inequality problems (9) and (10).

In the case of LTI systems, a necessary and sufficient condition has been established in Theorem 2.4.1 in [23] and Theorem 1.14 in [22] to guarantee Objectives 1 and 2. We extend those results to LPV systems in the following theorem. 
Theorem 8. Suppose that Assumptions 3, 4, and 5 are held. The closed-loop system (13) associated with the augmented multiagent system (7) and the augmented controller (12) with the state-space matrices given in (19) satisfies Objectives 1 and 2 if there exist matrices $\Pi$ and $\boldsymbol{\Gamma}(\rho(t))$ that satisfy the following algebraic equations:

$$
\begin{aligned}
\mathbf{A}(\rho(t)) \boldsymbol{\Pi}+\mathbf{B} \boldsymbol{\Gamma}(\rho(t))+\mathbf{E}(\rho(t)) & =\mathbf{\Pi} \mathbf{S}(\rho(t)) \\
\mathbf{C}^{e}(\rho(t)) \boldsymbol{\Pi}+\mathbf{D}^{e} \boldsymbol{\Gamma}(\rho(t))+\mathbf{F}^{e}(\rho(t)) & =\mathbf{0} .
\end{aligned}
$$

Proof. Suppose that matrices $\Pi$ and $\boldsymbol{\Gamma}(\rho(t))$ are solutions to (20a) and (20b). Defining $\widehat{\mathbf{A}}_{\mathrm{cl}}(\rho(t))$, as

$$
\widehat{\mathbf{A}}_{\mathrm{cl}}(\rho(t))=\left[\begin{array}{ccc}
\mathbf{I} & \mathbf{0} & \mathbf{0} \\
-\mathbf{I} & \mathbf{I} & \mathbf{0} \\
\mathbf{0} & \mathbf{0} & \mathbf{I}
\end{array}\right] \mathbf{A}_{\mathrm{cl}}(\rho(t))\left[\begin{array}{lll}
\mathbf{I} & \mathbf{0} & \mathbf{0} \\
\mathbf{I} & \mathbf{I} & \mathbf{0} \\
\mathbf{0} & \mathbf{0} & \mathbf{I}
\end{array}\right]
$$

results in the following, through some algebraic manipulations:

$$
\begin{aligned}
\widehat{\mathbf{A}}_{\mathrm{cl}}(\rho(t)) & =\left[\begin{array}{ccc}
\mathbf{A}(\rho(t))+\mathbf{B K}_{x} & \mathbf{B K}_{x} & \mathbf{B K}_{d}(\rho(t)) \\
\mathbf{0} & \mathbf{A}(\rho(t))+\mathbf{L}_{1} \mathbf{C}(\rho(t)) & \mathbf{E}(\rho(t))+\mathbf{L}_{1} \mathbf{F}(\rho(t)) \\
\mathbf{0} & \mathbf{L}_{2} \mathbf{C}(\rho(t)) & \mathbf{S}(\rho(t))+\mathbf{L}_{2} \mathbf{F}(\rho(t))
\end{array}\right] \\
& =\left[\begin{array}{ccc}
\mathbf{A}(\rho(t))+\mathbf{B K}_{x} & {\left[\mathbf{B K}_{x}\right.} & \left.\mathbf{B K}_{d}(\rho(t))\right] \\
\mathbf{0} & \mathbf{A}_{L}(\rho(t))
\end{array}\right],
\end{aligned}
$$

with $\mathbf{A}_{L}$ defined in (11). Due to the structure of $\widehat{\mathbf{A}}_{\mathrm{cl}}$ (that implies that it is asymptotically stable), there exists a symmetric positive definite matrix $\widehat{\mathbf{Q}}$ such that $\widehat{\mathbf{Q}} \widehat{\mathbf{A}}_{\mathrm{cl}}^{T}(\rho(t))+$ $\widehat{\mathbf{A}}_{\mathrm{cl}}(\rho(t)) \widehat{\mathbf{Q}}<\mathbf{0}$. On the other hand, there exists a symmetric positive definite matrix $\mathbf{Q}$ such that $\mathbf{Q} \mathbf{A}_{\mathrm{cl}}^{T}(\rho(t))+\mathbf{A}_{\mathrm{cl}}(\rho(t)) \mathbf{Q}<$ 0. This implies that the controller with the state-space matrices given by (19) satisfies Objective 1. Define $\mathbf{Y}=\operatorname{col}(\boldsymbol{\Pi}, \mathbf{I})$. Postmultiplying $\mathbf{A}^{c}$ by $\boldsymbol{\Upsilon}$ results in

$$
\begin{aligned}
\mathbf{A}^{c}(\rho(t)) \mathbf{\Upsilon} & \\
= & {\left[\begin{array}{c}
\mathbf{A}(\rho(t)) \boldsymbol{\Pi}+\mathbf{E}(\rho(t))+\mathbf{B} \boldsymbol{\Gamma}(\rho(t)) \\
\mathbf{S}(\rho(t))
\end{array}\right] } \\
& +\left[\begin{array}{l}
\mathbf{L}_{1} \\
\mathbf{L}_{2}
\end{array}\right](\mathbf{C}(\rho(t)) \boldsymbol{\Pi}+\mathbf{F}(\rho(t))+\mathbf{D} \boldsymbol{\Gamma}(\rho(t))) .
\end{aligned}
$$

Employing (19) and (20a) results in

$$
\begin{aligned}
& \mathbf{A}^{c}(\rho(t)) \mathbf{Y} \\
& =\left[\begin{array}{c}
\boldsymbol{\Pi} \\
\mathbf{I}
\end{array}\right] \mathbf{S}(\rho(t)) \\
& \quad-\mathbf{B}^{c}(\rho(t))(\mathbf{C}(\rho(t)) \boldsymbol{\Pi}+\mathbf{F}(\rho(t))+\mathbf{D} \Gamma(\rho(t))),
\end{aligned}
$$

which gives (16b). According to Lemma $7, \lim _{t \rightarrow \infty} \mathbf{e}(t)=\mathbf{0}$, and this concludes the proof.

\section{Output Regulation for Multiagent Polytopic LPV Systems}

To satisfy Objectives 1 and 2, (20a) and (20b) in Theorem 8 are needed to be solved. These equations are time-varying, which in principle provide an infinite number of linear equations. Neural networks have been employed to solve time-varying
Sylvester equation (20a) and (20b), but the upper bound on the convergence has been shown to be conservative [20]. To convert the problem to a set of finite number of linear equations, we restrict our study to polytopic LPV systems. Assume that time-varying matrices of system (1) have a polytopic dependency on the scheduling variables as

$$
\boldsymbol{\Omega}_{k}\left(\rho_{k}(t)\right)=\sum_{i=1}^{p_{k}} \rho_{k}^{i}(t) \boldsymbol{\Omega}_{k}^{i}, \quad \sum_{i=1}^{p_{k}} \rho_{k}^{i}(t)=1,
$$

where $\rho_{k}^{i}(t)$ are nonzero and nonnegative continuous functions, for $k \in \mathcal{N}$ and $\boldsymbol{\Omega} \in\left\{\mathbf{A}, \mathbf{C}, \mathbf{E}, \mathbf{F}, \mathbf{C}^{e}, \mathbf{F}^{e}, \mathbf{S}\right\}$. In addition, $\rho_{k}^{i}(t)$ represents the $i$ th element of the vector of scheduling variables $\rho_{k}(t)$.

Corollary 9. Suppose that Assumptions 3, 4, and 5 are held. The closed-loop system (13) associated with the augmented multiagent system (7) with the polytopic structure (25) and augmented controller (12) with the state-space matrices given in (19) satisfies Objectives 1 and 2 if there exist matrices $\Pi_{k}, \Gamma_{k}^{1}, \ldots, \Gamma_{k}^{p_{k}}$ that satisfy (26) for any $k \in N$

$$
\begin{aligned}
& {\left[\begin{array}{cccc}
\left(-\mathbf{S}_{k}^{1}\right) \oplus \mathbf{A}_{k}^{1} & \mathbf{I}_{n_{d_{k}}} \otimes \mathbf{B}_{k} & \cdots & \mathbf{0} \\
\mathbf{I}_{n_{d_{k}}} \otimes \mathbf{C}_{k}^{e^{1}} & \mathbf{I}_{n_{d_{k}}} \otimes \mathbf{D}_{k}^{e^{1}} & \cdots & \mathbf{0} \\
\vdots & \vdots & \ddots & \vdots \\
\left(-\mathbf{S}_{k}^{p_{k}}\right) \oplus \mathbf{A}_{k}^{p_{k}} & \mathbf{0} & \cdots & \mathbf{I}_{n_{d_{k}}} \otimes \mathbf{B}_{k} \\
\mathbf{I}_{n_{d_{k}}} \otimes \mathbf{C}_{k}^{e^{p_{k}}} & \mathbf{0} & \cdots & \mathbf{I}_{n_{d_{k}}} \otimes \mathbf{D}_{k}^{e^{p_{k}}}
\end{array}\right]\left[\begin{array}{c}
\operatorname{vec}\left(\boldsymbol{\Pi}_{k}\right) \\
\operatorname{vec}\left(\mathbf{\Gamma}_{k}^{1}\right) \\
\vdots \\
\operatorname{vec}\left(\mathbf{\Gamma}_{k}^{p_{k}}\right)
\end{array}\right]} \\
& =\left[\begin{array}{c}
-\operatorname{vec}\left(\mathbf{E}_{k}^{1}\right) \\
-\operatorname{vec}\left(\mathbf{F}_{k}^{e^{1}}\right) \\
\vdots \\
-\operatorname{vec}\left(\mathbf{E}_{k}^{p_{k}}\right) \\
-\operatorname{vec}\left(\mathbf{F}_{k}^{p_{k}}\right)
\end{array}\right] .
\end{aligned}
$$


Proof. Assume that $\Pi$ and $\Gamma(\rho(t))$ are constructed by using $\Pi=\operatorname{diag}\left(\boldsymbol{\Pi}_{1}, \ldots, \boldsymbol{\Pi}_{N}\right)$ and $\boldsymbol{\Gamma}(\rho(t))=\operatorname{diag}\left(\boldsymbol{\Gamma}_{1}(\rho(t)), \ldots\right.$, $\left.\boldsymbol{\Gamma}_{N}(\rho(t))\right)$. Due to the structure of the state-space matrices of the augmented system (7), (20a) and (20b) are equivalent with

$$
\begin{aligned}
& \mathbf{A}_{k}\left(\rho_{k}(t)\right) \boldsymbol{\Pi}_{k}+\mathbf{B}_{k} \boldsymbol{\Gamma}_{k}\left(\rho_{k}(t)\right)+\mathbf{E}_{k}\left(\rho_{k}(t)\right) \\
& \quad=\boldsymbol{\Pi}_{k} \mathbf{S}_{k}\left(\rho_{k}(t)\right), \\
& \mathbf{C}_{k}^{e}\left(\rho_{k}(t)\right) \boldsymbol{\Pi}_{k}+\mathbf{D}_{k}^{e} \boldsymbol{\Gamma}_{k}\left(\rho_{k}(t)\right)+\mathbf{F}_{k}^{e}\left(\rho_{k}(t)\right)=\mathbf{0},
\end{aligned}
$$

for any $k \in N$. We consider that $\Gamma_{k}\left(\rho_{k}(t)\right)$ has a polytopic structure as in (25); that is, $\boldsymbol{\Gamma}_{k}\left(\rho_{k}(t)\right)=\sum_{i=1}^{p_{k}} \rho_{k}^{i}(t) \boldsymbol{\Gamma}_{k}^{i}$, for $k \epsilon$ $N$. The following equations are obtained by substituting (25) into (27):

$$
\begin{aligned}
& \sum_{i=1}^{p_{k}} \rho_{k}^{i}(t) \mathbf{A}_{k}^{i} \boldsymbol{\Pi}_{k}+\mathbf{B}_{k} \sum_{i=1}^{p_{k}} \rho_{k}^{i}(t) \boldsymbol{\Gamma}_{k}^{i}+\sum_{i=1}^{p_{k}} \rho_{k}^{i}(t) \mathbf{E}_{k}^{i} \\
& =\boldsymbol{\Pi}_{k} \sum_{i=1}^{p_{k}} \rho_{k}^{i}(t) \mathbf{S}_{k}^{i} \\
& \sum_{i=1}^{p_{k}} \rho_{k}^{i}(t) \mathbf{C}_{k}^{e^{i}} \boldsymbol{\Pi}_{k}+\mathbf{D}_{k}^{e} \sum_{i=1}^{p_{k}} \rho_{k}^{i}(t) \boldsymbol{\Gamma}_{k}^{i}+\sum_{i=1}^{p_{k}} \rho_{k}^{i}(t) \mathbf{F}_{k}^{e^{i}}=\mathbf{0},
\end{aligned}
$$

and hence

$$
\begin{aligned}
\sum_{i=1}^{p_{k}} \rho_{k}^{i}(t)\left(\mathbf{A}_{k}^{i} \boldsymbol{\Pi}_{k}+\mathbf{B}_{k} \boldsymbol{\Gamma}_{k}^{i}+\mathbf{E}_{k}^{i}-\boldsymbol{\Pi}_{k} \mathbf{S}_{k}^{i}\right) & =\mathbf{0}, \\
\sum_{i=1}^{p_{k}} \rho_{k}^{i}(t)\left(\mathbf{C}_{k}^{e^{i}} \boldsymbol{\Pi}_{k}+\mathbf{D}_{k}^{e} \boldsymbol{\Gamma}_{k}^{i}+\mathbf{F}_{k}^{e^{i}}\right) & =\mathbf{0} .
\end{aligned}
$$

Since $\rho_{k}^{i}(t)$ are nonzero and nonnegative continuous functions for $k \in N$ and $i \in\left\{1, \ldots, p_{k}\right\}$, the following equations are concluded from (29):

$$
\begin{aligned}
\mathbf{A}_{k}^{i} \boldsymbol{\Pi}_{k}+\mathbf{B}_{k} \Gamma_{k}^{i}+\mathbf{E}_{k}^{i} & =\boldsymbol{\Pi}_{k} \mathbf{S}_{k}^{i}, \\
\mathbf{C}_{k}^{e^{i}} \boldsymbol{\Pi}_{k}+\mathbf{D}_{k}^{e} \boldsymbol{\Gamma}_{k}^{i}+\mathbf{F}_{k}^{e^{i}} & =\mathbf{0},
\end{aligned}
$$

for any $k \in N$ and $i \in\left\{1, \ldots, p_{k}\right\}$. Equations (30a) and (30b) can be rewritten in the following forms by applying the Kronecker product notation and the vectorization operator:

$$
\begin{aligned}
& \left(\left(\mathbf{I}_{n_{k}^{d}} \otimes \mathbf{A}_{k}^{i}\right)-\left(\mathbf{S}_{k}^{i} \otimes \mathbf{I}_{n_{k}^{x}}\right)\right) \operatorname{vec}\left(\boldsymbol{\Pi}_{k}\right) \\
& \quad+\left(\mathbf{I}_{n_{k}^{d}} \otimes \mathbf{B}_{k}\right) \operatorname{vec}\left(\boldsymbol{\Gamma}_{k}^{i}\right)=-\operatorname{vec}\left(\mathbf{E}_{k}^{i}\right), \\
& \left(\mathbf{I}_{n_{k}^{d}} \otimes \mathbf{C}_{k}^{e^{i}}\right) \operatorname{vec}\left(\boldsymbol{\Pi}_{k}\right) \\
& \quad+\left(\mathbf{I}_{n_{k}^{d}} \otimes \mathbf{D}_{k}^{e^{i}}\right) \operatorname{vec}\left(\boldsymbol{\Gamma}_{k}^{i}\right)=-\operatorname{vec}\left(\mathbf{F}_{k}^{e^{i}}\right),
\end{aligned}
$$

for any $k \in N$ and $i \in\left\{1, \ldots, p_{k}\right\}$. Finally, for any $k \in N,(26)$ is obtained by combining the $p_{k}$ equations in (31), and this concludes the proof.

Lemma 10 provides a simple condition to guarantee the solvability of (26).
Lemma 10. Consider that Assumptions 3, 4, and 5 are held. Equation (26) has a solution if

$$
n_{e_{k}}+\frac{p_{k}-1}{p_{k}} n_{x_{k}} \leq n_{u_{k}}
$$

for any $k \in \mathcal{N}$.

Proof. The number of equations and the number of free variables in linear equation (26) are $n_{d_{k}} p_{k}\left(n_{x_{k}}+n_{e_{k}}\right)$ and $n_{d_{k}}\left(n_{x_{k}}+p_{k} n_{u_{k}}\right)$, respectively. The block rows of (26) are linearly independent. If inequality $n_{d_{k}} p_{k}\left(n_{x_{k}}+n_{e_{k}}\right) \leq n_{d_{k}}\left(n_{x_{k}}+\right.$ $p_{k} n_{u_{k}}$ ) is satisfied, then the system of linear equations (26) has a solution. The latter condition results in inequality (32).

Remark 11. In the case of LTI agents, where $p_{k}=1$ for $k \in N$, inequality (32) becomes $n_{e_{k}} \leq n_{u_{k}}$, which is independent of $n_{x_{k}}$. This has been shown in $[22,24]$.

The proposed control design method can be summarized as follows. First, matrices $\mathbf{K}_{x}$ and $\operatorname{col}\left(\mathbf{L}_{1}, \mathbf{L}_{2}\right)$ are determined to satisfy Assumptions 3 and 5 by solving the matrix inequality problems given in (9) and (10), respectively. Second, matrices $\Pi_{k}, \Gamma_{k}^{1}, \ldots, \Gamma_{k}^{p_{k}}$ and $\boldsymbol{\Gamma}_{k}\left(\rho_{k}(t)\right)=\sum_{i=1}^{p_{k}} \rho_{k}^{i}(t) \boldsymbol{\Gamma}_{k}^{i}$ are obtained by solving equation (26), for $k \in \mathcal{N}$. Then, the statespace matrices of the controller are obtained by substituting the augmented matrices $\mathbf{K}_{x}, \operatorname{col}\left(\mathbf{L}_{1}, \mathbf{L}_{2}\right), \boldsymbol{\Pi}$, and $\boldsymbol{\Gamma}$ in (12).

\section{Illustrative Examples}

In this section, two numerical examples are given to illustrate the efficacy of the proposed cooperative control design method of this paper.

Example 1. Consider a group of 4 agents modeled as secondorder LPV polytopic systems dependent on three scheduling variables. The state-space matrices of the agents and the exosystem are generated in MATLAB as follows:

$$
\begin{aligned}
& \mathbf{A}_{1}^{1}=\left[\begin{array}{ll}
-1 & 6 \\
-7 & 0
\end{array}\right], \\
& \mathbf{A}_{1}^{2}=\left[\begin{array}{cc}
-2 & 7 \\
-5 & -4
\end{array}\right], \\
& \mathbf{A}_{1}^{3}=\left[\begin{array}{cc}
5 & -1 \\
-3 & 0
\end{array}\right], \\
& \mathbf{C}_{1}^{1}=\left[\begin{array}{cc}
8 & 2 \\
-1 & -3
\end{array}\right], \\
& \mathbf{C}_{1}^{2}=\left[\begin{array}{cc}
8 & 1 \\
-3 & -5
\end{array}\right], \\
& \mathbf{C}_{1}^{3}=\left[\begin{array}{cc}
7 & 10 \\
9 & -9
\end{array}\right],
\end{aligned}
$$




$$
\begin{aligned}
& \mathbf{C}_{1}^{e^{1}}=\left[\begin{array}{ll}
-1 & 7
\end{array}\right], \\
& \mathbf{C}_{1}^{e^{2}}=\left[\begin{array}{ll}
4 & 7
\end{array}\right] \text {, } \\
& \mathbf{C}_{1}^{e^{3}}=\left[\begin{array}{ll}
0 & 8
\end{array}\right] \text {, } \\
& \mathbf{E}_{1}^{1}=\left[\begin{array}{cc}
7 & -9 \\
3 & 6
\end{array}\right] \text {, } \\
& \mathbf{E}_{1}^{2}=\left[\begin{array}{cc}
-2 & -5 \\
2 & 0
\end{array}\right] \text {, } \\
& \mathbf{E}_{1}^{3}=\left[\begin{array}{rr}
-1 & 4 \\
10 & 7
\end{array}\right] \text {, } \\
& \mathbf{F}_{1}^{1}=\left[\begin{array}{cc}
-7 & 7 \\
8 & 6
\end{array}\right] \text {, } \\
& \mathbf{F}_{1}^{2}=\left[\begin{array}{cc}
-4 & -8 \\
8 & 6
\end{array}\right] \text {, } \\
& \mathbf{F}_{1}^{3}=\left[\begin{array}{ll}
-9 & -5 \\
-3 & -3
\end{array}\right] \text {, } \\
& \mathbf{F}_{1}^{e^{1}}=\left[\begin{array}{ll}
-8 & -7
\end{array}\right] \text {, } \\
& \mathbf{F}_{1}^{e^{2}}=\left[\begin{array}{ll}
-4 & 4
\end{array}\right] \text {, } \\
& \mathbf{F}_{1}^{e^{3}}=\left[\begin{array}{ll}
-5 & 4
\end{array}\right] \text {, } \\
& \mathbf{B}_{1}=\left[\begin{array}{ccc}
-4 & -1 & 10 \\
9 & 1 & -4
\end{array}\right] \text {, } \\
& \mathbf{D}_{1}=\left[\begin{array}{lll}
-2 & 7 & -1 \\
-7 & 0 & -8
\end{array}\right] \text {, } \\
& \mathbf{D}_{1}^{e}=\left[\begin{array}{lll}
-9 & 1 & -9
\end{array}\right] \text {, } \\
& \mathbf{S}_{1}^{1}=\left[\begin{array}{cc}
0 & -10 \\
10 & 0
\end{array}\right] \text {, } \\
& \mathbf{S}_{1}^{2}=\left[\begin{array}{cc}
0 & -10 \\
10 & 0
\end{array}\right] \text {, } \\
& \mathbf{S}_{1}^{3}=\left[\begin{array}{cc}
0 & -5 \\
5 & 0
\end{array}\right] \text {. }
\end{aligned}
$$

We assume that the vertices of the matrices for all the agents are the same, that is, $\boldsymbol{\Omega}_{m}^{i}=\boldsymbol{\Omega}_{1}^{i}$ and $\boldsymbol{\Lambda}_{m}=\boldsymbol{\Lambda}_{1}$, for $m \in$ $\{2,3,4\}$ and $i \in\{1,2,3\}$, where $\boldsymbol{\Omega} \in\left\{\mathbf{A}, \mathbf{C}, \mathbf{E}, \mathbf{F}, \mathbf{C}^{e}, \mathbf{F}^{e}, \mathbf{S}\right\}$ and $\boldsymbol{\Lambda} \in\left\{\mathbf{B}, \mathbf{D}, \mathbf{D}^{e}\right\}$. We consider sinusoidal disturbance inputs, as given in $[14,15,24]$. In addition, the following trajectories are considered for the scheduling variables:

$$
\rho_{k}^{i}(t)=\alpha_{k}^{i}\left(1-\sin \left(\omega_{k}^{i} t\right)\right)
$$

where $\alpha_{k}^{i} \leq 1 / 6$ and $\omega_{k}^{i} \leq 1$ are randomly generated positive numbers for $k \in\{1,2,3,4\}$ and $i \in\{1,2\}$ and $\rho_{k}^{3}(t)=$ $1-\sum_{i=1}^{2} \rho_{k}^{i}(t)$. Matrices $\mathbf{K}_{x}$ and $\operatorname{col}\left(\mathbf{L}_{1}, \mathbf{L}_{2}\right)$ are determined to satisfy Assumptions 3 and 5 by solving the matrix inequality problems given in (9) and (10), respectively. These matrices are determined to be

$$
\begin{gathered}
\mathbf{K}_{x}=\operatorname{diag}\left(\left[\begin{array}{ll}
3 & 2
\end{array}\right],\left[\begin{array}{ll}
3 & 2
\end{array}\right],\left[\begin{array}{ll}
3 & 2
\end{array}\right],\left[\begin{array}{ll}
3 & 2
\end{array}\right]\right), \\
{\left[\begin{array}{l}
\mathbf{L}_{1} \\
\mathbf{L}_{2}
\end{array}\right]=\operatorname{diag}\left(\left[\begin{array}{cc}
1.2961 & -0.5672 \\
-0.1216 & -4.971 \\
-0.1209 & 0.0641 \\
0.0952 & -0.408
\end{array}\right],\right.} \\
{\left[\begin{array}{cc}
1.2961 & -0.5672 \\
-0.1216 & -4.971 \\
-0.1209 & 0.0641 \\
0.0952 & -0.408
\end{array}\right],\left[\begin{array}{cc}
1.2961 & -0.5672 \\
-0.1216 & -4.971 \\
-0.1209 & 0.0641 \\
0.0952 & -0.408
\end{array}\right],} \\
\left.\left[\begin{array}{cc}
1.2961 & -0.5672 \\
-0.1216 & -4.971 \\
-0.1209 & 0.0641 \\
0.0952 & -0.408
\end{array}\right]\right)
\end{gathered}
$$

Since inequality (32) in Lemma 10 is satisfied, (26) has a solution determined to be

$$
\begin{aligned}
& \Pi_{k}=\operatorname{diag}\left(\left[\begin{array}{cc}
-1.0109 & -2.4683 \\
-2.0822 & 1.6170
\end{array}\right],\right. \\
& {\left[\begin{array}{ll}
-1.0109 & -2.4683 \\
-2.0822 & 1.6170
\end{array}\right],\left[\begin{array}{cc}
-1.0109 & -2.4683 \\
-2.0822 & 1.6170
\end{array}\right],} \\
& \left.\left[\begin{array}{ll}
-1.0109 & -2.4683 \\
-2.0822 & 1.6170
\end{array}\right]\right) \\
& \Gamma_{k}^{1}=\operatorname{diag}\left(\left[\begin{array}{cc}
-0.2685 & 0.0431 \\
0 & 0 \\
-2.1275 & 0.7111
\end{array}\right],\right.
\end{aligned}
$$$$
\left[\begin{array}{cc}
-0.2685 & 0.0431 \\
0 & 0 \\
-2.1275 & 0.7111
\end{array}\right],\left[\begin{array}{cc}
-0.2685 & 0.0431 \\
0 & 0 \\
-2.1275 & 0.7111
\end{array}\right],
$$$$
\left.\left[\begin{array}{cc}
-0.2685 & 0.0431 \\
0 & 0 \\
-2.1275 & 0.7111
\end{array}\right]\right)
$$ 


$$
\begin{aligned}
& \Gamma_{k}^{2}=\operatorname{diag}\left(\left[\begin{array}{cc}
-0.4412 & 1.9580 \\
7.9419 & 18.1930 \\
-1.1895 & 0.6685
\end{array}\right]\right. \text {, } \\
& {\left[\begin{array}{cc}
-0.4412 & 1.9580 \\
7.9419 & 18.1930 \\
-1.1895 & 0.6685
\end{array}\right],\left[\begin{array}{cc}
-0.4412 & 1.9580 \\
7.9419 & 18.1930 \\
-1.1895 & 0.6685
\end{array}\right] \text {, }} \\
& \left.\left[\begin{array}{cc}
-0.4412 & 1.9580 \\
7.9419 & 18.1930 \\
-1.1895 & 0.6685
\end{array}\right]\right) \\
& \Gamma_{k}^{3}=\operatorname{diag}\left(\left[\begin{array}{cc}
-1.1210 & 0.2718 \\
0 & 0 \\
-1.2853 & 1.6100
\end{array}\right]\right. \text {, } \\
& {\left[\begin{array}{cc}
-1.1210 & 0.2718 \\
0 & 0 \\
-1.2853 & 1.6100
\end{array}\right],\left[\begin{array}{cc}
-1.1210 & 0.2718 \\
0 & 0 \\
-1.2853 & 1.6100
\end{array}\right],} \\
& \left.\left[\begin{array}{cc}
-1.1210 & 0.2718 \\
0 & 0 \\
-1.2853 & 1.6100
\end{array}\right]\right) \text {, }
\end{aligned}
$$

where $\boldsymbol{\Gamma}_{k}(\rho(t))=\sum_{i=1}^{3} \rho_{k}^{i}(t) \boldsymbol{\Gamma}_{k}^{i}$. Objectives 1 and 2 are met by controller (12) whose state-space matrices are calculated using (19). The tracking errors for the closed-loop system using the designed controllers are shown in Figure 1 and guaranteed to be asymptotically stable with arbitrary initial conditions for agents and exogenous systems. The control actions that the agents' controllers provide are shown in Figure 2.

Example 2. Consider a group of 6 agents modeled as secondorder polytopic LPV systems dependent on 4 scheduling variables. The state-space matrices of the agents and the exosystem are generated in MATLAB as follows:

$$
\begin{aligned}
& \mathbf{A}_{1}^{1}=\left[\begin{array}{cc}
-5 & 6 \\
-3 & -2
\end{array}\right], \\
& \mathbf{A}_{1}^{2}=\left[\begin{array}{cc}
-6 & -3 \\
-1 & 2
\end{array}\right], \\
& \mathbf{A}_{1}^{3}=\left[\begin{array}{ll}
-1 & -3 \\
6 & -10
\end{array}\right], \\
& \mathbf{A}_{1}^{4}=\left[\begin{array}{ll}
-5 & 0 \\
-10 & -4
\end{array}\right], \\
& \mathbf{C}_{1}^{1}=\left[\begin{array}{ll}
0 & -8
\end{array}\right], \\
& \mathbf{C}_{1}^{2}=\left[\begin{array}{ll}
0 & -6
\end{array}\right],
\end{aligned}
$$

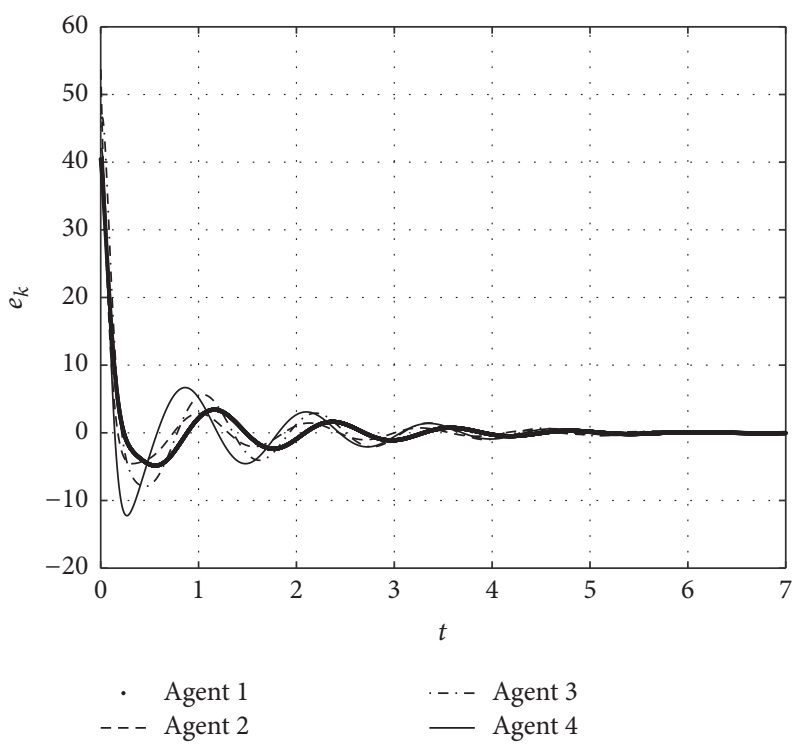

FIGURE 1: Regulated outputs of four agents for Example 1.
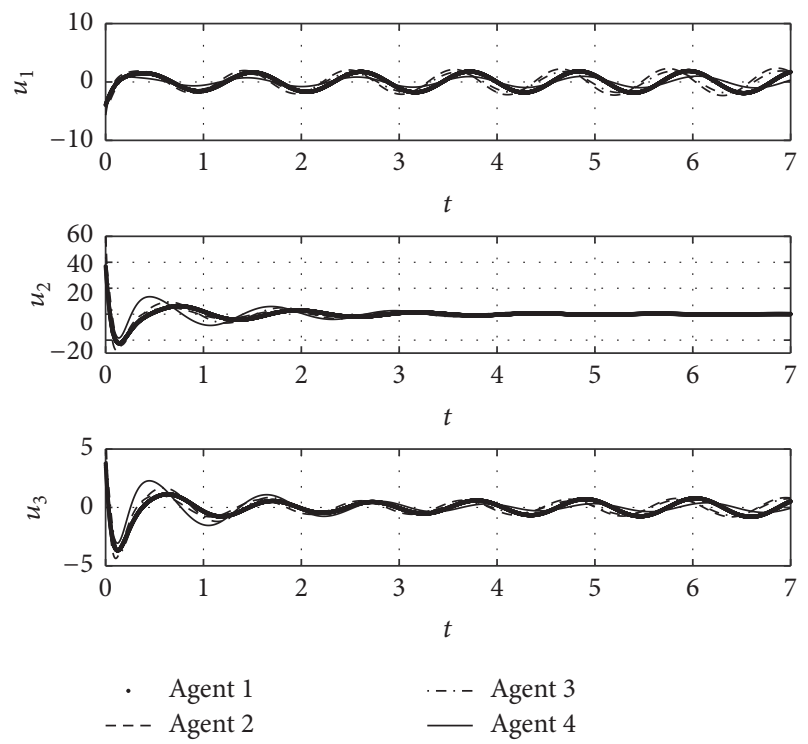

FIGURE 2: Control inputs to the four agents for Example 1.

$$
\begin{aligned}
\mathbf{C}_{1}^{3} & =\left[\begin{array}{ll}
3 & -8
\end{array}\right], \\
\mathbf{C}_{1}^{4} & =\left[\begin{array}{ll}
2 & -8
\end{array}\right], \\
\mathbf{C}_{1}^{e^{1}} & =\left[\begin{array}{ll}
2 & 1
\end{array}\right], \\
\mathbf{C}_{1}^{e^{2}} & =\left[\begin{array}{ll}
0 & -7
\end{array}\right], \\
\mathbf{C}_{1}^{e^{3}} & =\left[\begin{array}{ll}
10 & 8
\end{array}\right], \\
\mathbf{C}_{1}^{e^{4}} & =\left[\begin{array}{ll}
-5 & -3
\end{array}\right], \\
\mathbf{E}_{1}^{1} & =\left[\begin{array}{ll}
0 & 2 \\
4 & 6
\end{array}\right],
\end{aligned}
$$




$$
\begin{aligned}
& \mathbf{E}_{1}^{2}=\left[\begin{array}{cc}
-7 & -4 \\
7 & 10
\end{array}\right], \\
& \mathbf{E}_{1}^{3}=\left[\begin{array}{cc}
-8 & 7 \\
9 & 9
\end{array}\right], \\
& \mathbf{E}_{1}^{4}=\left[\begin{array}{cc}
-5 & -7 \\
-7 & 7
\end{array}\right], \\
& \mathbf{F}_{1}^{1}=\left[\begin{array}{ll}
2 & 7
\end{array}\right], \\
& \mathbf{F}_{1}^{2}=\left[\begin{array}{ll}
5 & -3
\end{array}\right], \\
& \mathbf{F}_{1}^{3}=\left[\begin{array}{ll}
0 & 0
\end{array}\right], \\
& \mathbf{F}_{1}^{4}=\left[\begin{array}{ll}
3 & -9
\end{array}\right], \\
& \mathbf{F}_{1}^{e^{1}}=\left[\begin{array}{ll}
-4 & -1
\end{array}\right], \\
& \mathbf{F}_{1}^{e^{2}}=\left[\begin{array}{ll}
-7 & 0
\end{array}\right], \\
& \mathbf{F}_{1}^{e^{3}}=\left[\begin{array}{ll}
-4 & 9
\end{array}\right], \\
& \mathbf{F}_{1}^{e^{4}}=\left[\begin{array}{ll}
9 & 5
\end{array}\right], \\
& \mathbf{B}_{1}=\left[\begin{array}{l}
2 \\
4
\end{array}\right], \\
& \mathbf{D}_{1}=\left[\begin{array}{ll}
6
\end{array}\right], \\
& \mathbf{D}_{1}^{e}=\left[\begin{array}{ll}
9
\end{array}\right], \\
& \mathbf{S}_{1}^{1}=\mathbf{S}_{1}^{3}=\mathbf{S}_{1}^{4}=\left[\begin{array}{ll}
0 & -7 \\
7 & 0
\end{array}\right], \\
& \mathbf{S}_{1}^{2}=\left[\begin{array}{ll}
0 & -2 \\
2 & 0
\end{array}\right]
\end{aligned}
$$

Similar to Example 1, we assume that the vertices of all the agents' matrices are the same. In addition, the scheduling variables are continuous functions as

$$
\rho_{k}^{i}(t)=\alpha_{k}^{i}\left(1-\sin \left(\omega_{k}^{i} t\right)\right)
$$

where $\alpha_{k}^{i} \leq 1 / 8$ and $\omega_{k}^{i} \leq 1$ are randomly generated positive numbers for $k \in\{1,2,3,4,5,6\}$ and $i \in\{1,2,3\}$ and $\rho_{k}^{4}(t)=1-\sum_{i=1}^{3} \rho_{k}^{i}(t)$. Matrices $\mathbf{K}_{x}$ and $\operatorname{col}\left(\mathbf{L}_{1}, \mathbf{L}_{2}\right)$ satisfying Assumptions 3 and 5 are determined by solving the matrix inequality problems given in (9) and (10), respectively. In addition, $\boldsymbol{\Pi}$ and $\boldsymbol{\Gamma}(\rho(t))=\sum_{i=1}^{4} \rho_{k}^{i}(t) \boldsymbol{\Gamma}_{k}^{\mathrm{i}}$ are calculated by solving the linear equation (26). Objective 2 is satisfied by the controller (12) with the state-space matrices given by (19). Considering an arbitrary bounded initial condition for agents and the exogenous systems, the tracking errors associated with 6 agents are depicted in Figure 3. The figure illustrates that tracking is achieved by all the agents in the presence of external disturbances with agents dynamics affected by parameter variability.



FIgURE 3: Tracking errors for six agents of Example 2.

\section{Conclusion}

In this paper, we have addressed the cooperative output regulation problem for heterogeneous multiagent LPV systems. The parameter-varying controllers are designed assuming that the controllers are fully connected and exchange information while the agents are decoupled from each other. To design the controllers, a time-varying Sylvester equation needs to be solved, and the solution to it is obtained for the case, where the agents dynamics are described by polytopic LPV models. The future prospect of this work is to address the case of controllers' communication described by a spanning tree graph.

\section{Competing Interests}

The authors declare that there is no conflict of interests regarding the publication of this paper.

\section{References}

[1] Y. Cao, W. Yu, W. Ren, and G. Chen, "An overview of recent progress in the study of distributed multi-agent coordination," IEEE Transactions on Industrial Informatics, vol. 9, no. 1, pp. 427-438, 2013.

[2] K.-K. Oh, M.-C. Park, and H.-S. Ahn, "A survey of multi-agent formation control," Automatica, vol. 53, pp. 424-440, 2015.

[3] M. Mesbahi and M. Egerstedt, Graph Theoretic Methods in Multiagent Networks, Princeton Series in Applied Mathematics, Princeton University Press, Princeton, NJ, USA, 2010.

[4] L. Yu and J. Wang, "Cooperative control for uncertain multiagent systems via distributed output regulation," Mathematical Problems in Engineering, vol. 2013, Article ID 898375, 11 pages, 2013.

[5] X. Wang, Y. Hong, J. Huang, and Z.-P. Jiang, "A distributed control approach to a robust output regulation problem for 
multi-agent linear systems," IEEE Transactions on Automatic Control, vol. 55, no. 12, pp. 2891-2895, 2010.

[6] Y. Su and J. Huang, "Cooperative output regulation of linear multi-agent systems," IEEE Transactions on Automatic Control, vol. 57, no. 4, pp. 1062-1066, 2012.

[7] D. Huang, X. Wang, Y. Niu, and L. Shen, "Cooperative output regulation of nonlinear multiagent systems under a general directed graph," Mathematical Problems in Engineering, vol. 2015, Article ID 456121, 9 pages, 2015.

[8] J. Mohammadpour and C. Scherer, Control of Linear Parameter Varying Systems with Applications, Springer, New York, NY, USA, 2012.

[9] Y. Bolea, N. Chefdor, and A. Grau, "MIMO LPV state-space identification of open-flow irrigation canal systems," Mathematical Problems in Engineering, vol. 2012, Article ID 948936, 16 pages, 2012.

[10] O. Sename, P. Gaspar, and J. Bokor, Robust Control and Linear Parameter Varying Approaches, Springer, Berlin, Germany, 2013.

[11] G. Cao, K. M. Grigoriadis, and Y. D. Nyanteh, "LPV control for the full region operation of a wind turbine integrated with synchronous generator," The Scientific World Journal, vol. 2015, Article ID 638120, 15 pages, 2015.

[12] V. Ugrinovskii, "Gain-scheduled synchronization of uncertain parameter varying systems via relative $H_{\infty}$ consensus," in Proceedings of the 50th IEEE Conference on Decision and Control and European Control Conference (CDC-ECC '11), pp. 42514256, IEEE, Orlando, Fla, USA, December 2011.

[13] V. Ugrinovskii, "Gain-scheduled synchronization of parameter varying systems via relative $H_{\infty}$ consensus with application to synchronization of uncertain bilinear systems," Automatica, vol. 50, no. 11, pp. 2880-2887, 2014.

[14] G. S. Seyboth, G. S. Schmidt, and F. Allgower, "Output synchronization of linear parameter-varying systems via dynamic couplings," in Proceedings of the 51st IEEE Conference on Decision and Control (CDC '12), pp. 5128-5133, December 2012.

[15] G. S. Seyboth, G. S. Schmidt, and F. Allgower, "Cooperative control of linear parameter-varying systems," in Proceedings of the American Control Conference (ACC '12), pp. 2407-2412, June 2012.

[16] Y. Zhang, D. Jiang, and J. Wang, "A recurrent neural network for solving sylvester equation with time-varying coefficients," IEEE Transactions on Neural Networks, vol. 13, no. 5, pp. 1053-1063, 2002.

[17] S. Li and Y. Li, "Nonlinearly activated neural network for solving time-varying complex sylvester equation," IEEE Transactions on Cybernetics, vol. 44, no. 8, pp. 1397-1407, 2014.

[18] S. Li, S. Chen, and B. Liu, "Accelerating a recurrent neural network to finite-time convergence for solving time-varying sylvester equation by using a sign-Bi-power activation function," Neural Processing Letters, vol. 37, no. 2, pp. 189-205, 2013.

[19] L. Xiao and Y. Zhang, "From different zhang functions to various ZNN models accelerated to finite-time convergence for time-varying linear matrix equation," Neural Processing Letters, vol. 39, no. 3, pp. 309-326, 2014.

[20] Y. Shen, P. Miao, Y. Huang, and Y. Shen, "Finite-time stability and its application for solving time-varying sylvester equation by recurrent neural network," Neural Processing Letters, vol. 42, no. 3, pp. 763-784, 2015.

[21] P. Apkarian and R. J. Adams, "Advanced gain-scheduling techniques for uncertain systems," IEEE Transactions on Control Systems Technology, vol. 6, no. 1, pp. 21-32, 1998.
[22] J. Huang, Nonlinear Output Regulation, Society for Industrial and Applied Mathematics, 2004.

[23] A. Saberi, A. A. Stoorvogel, and P. Sannuti, Control of linear systems with regulation and input constraints, Communications and Control Engineering Series, Springer-Verlag, London, England, 2000.

[24] Y. Su and J. Huang, "Cooperative output regulation of linear multi-agent systems by output feedback," Systems \& Control Letters, vol. 61, no. 12, pp. 1248-1253, 2012. 


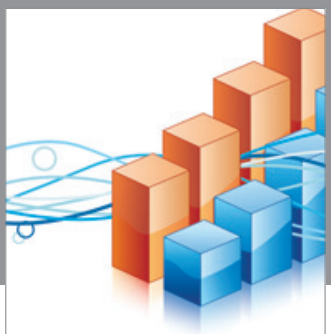

Advances in

Operations Research

vatem alat4

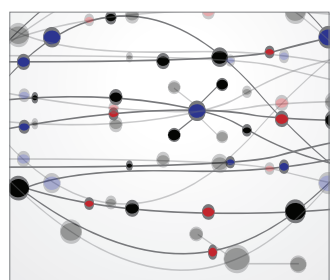

\section{The Scientific} World Journal
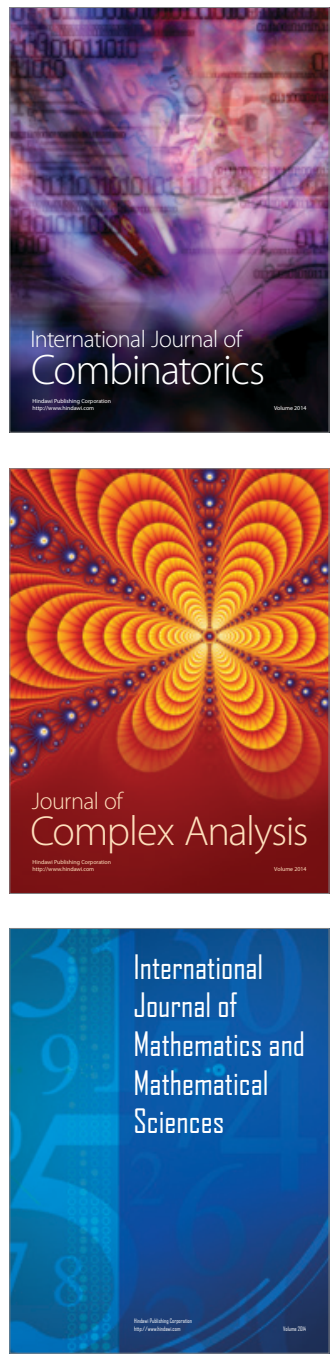
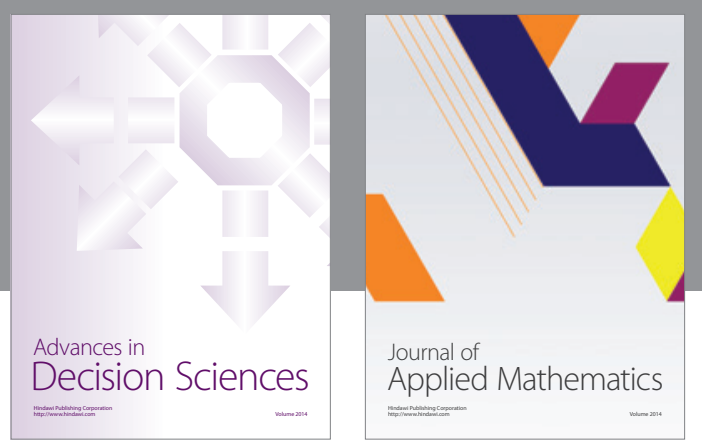

Algebra

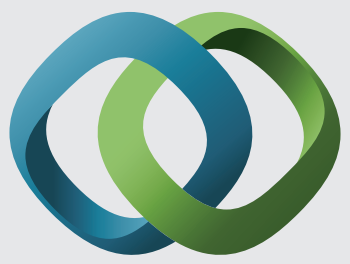

\section{Hindawi}

Submit your manuscripts at

https://www.hindawi.com
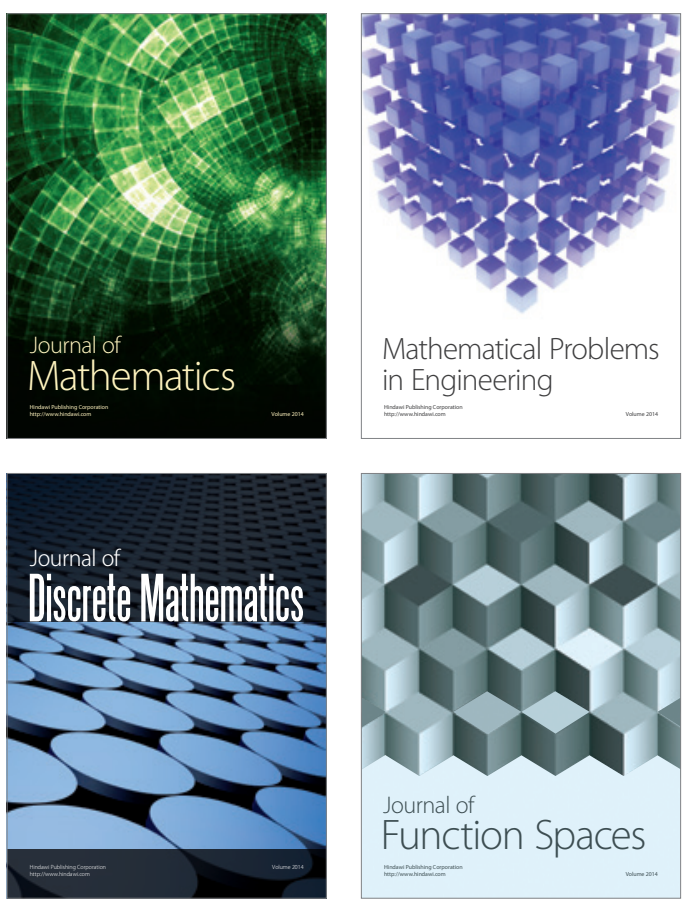

Mathematical Problems in Engineering
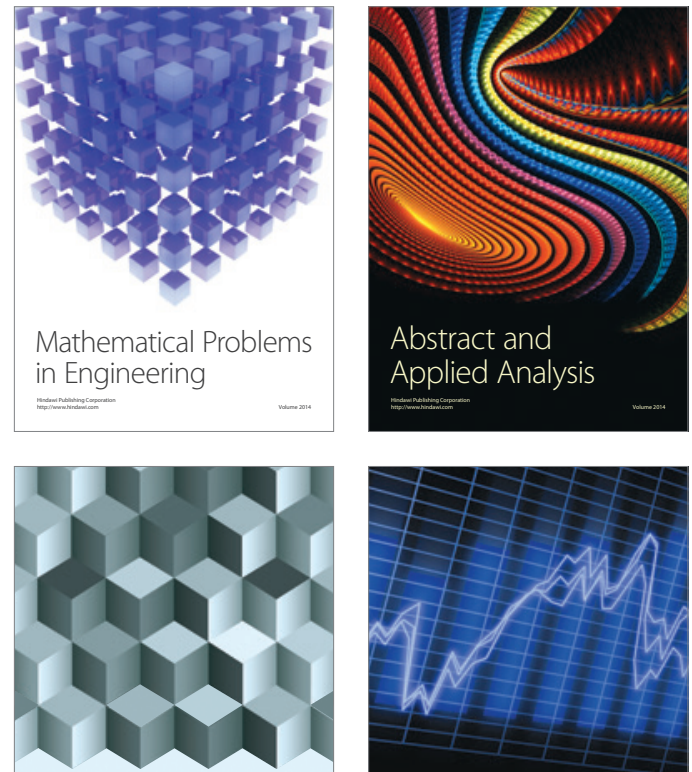

Journal of

Function Spaces

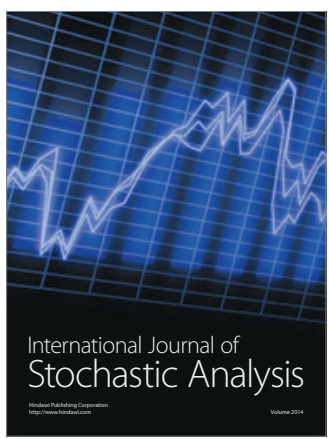

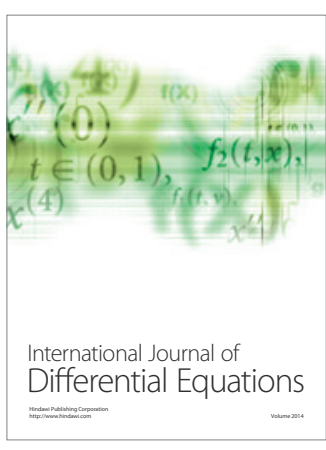
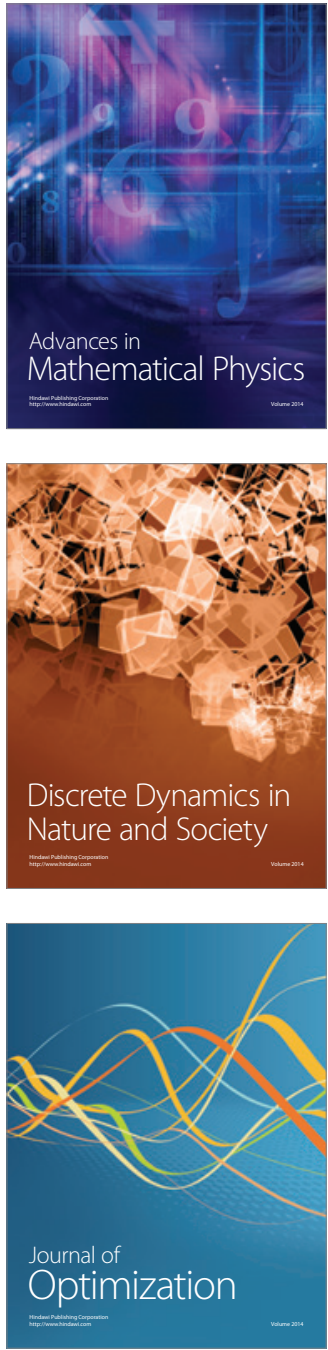